\title{
THE SYMMETRIC GENUS OF SPORADIC GROUPS
}

\author{
M. D. E. CONDER, R. A. WILSON, AND A. J. WOLDAR
}

(Communicated by Ronald N. Solomon)

\begin{abstract}
Given a finite group $G$, the symmetric genus of $G$ is defined to be the smallest integer $g$ such that $G$ acts faithfully on a closed orientable surface of genus $g$. Previous to this work, the task of determining the symmetric genus for the sporadic simple groups had been completed for all but nine groups: $J_{3}$, $\mathrm{McL}, \mathrm{Suz}, \mathrm{O}^{\prime} \mathrm{N}, \mathrm{Co}_{2}, \mathrm{Fi}_{23}, \mathrm{Co}_{1}, \mathrm{~B}$, and $\mathrm{M}$. In the present paper the authors resolve the problem for six of these groups, viz. $\mathrm{J}_{3}, \mathrm{McL}, \mathrm{Suz}, \mathrm{O}{ }^{\prime} \mathrm{N}, \mathrm{Co}_{2}$, and $\mathrm{Co}_{1}$. Significant progress is also reported for the group $\mathrm{Fi}_{23}$.
\end{abstract}

\section{INTRODUCTION}

This paper is intended as a sequel to our earlier paper [8], which formed the basis of a talk delivered by the third author at The Marshall Hall Conference on Group Theory, Design Theory, and Coding Theory at the University of Vermont in September 1990. The main purpose of this paper is to supply proofs conspicuously absent from the earlier paper due to constraints on its length.

The symmetric genus of a finite group $G$ (so defined by Tucker in [23]) is the smallest genus of all closed orientable surfaces on which $G$ acts faithfully as a group of automorphisms (i.e., homeomorphisms of the surface onto itself) allowing reversal of the surface's orientation. If we restrict ourselves to automorphisms that preserve orientation, we obtain what Tucker calls the strong symmetric genus of $G$. The latter is generally larger than the former, but in many instances the two are equal (for example, when $G$ has no subgroup of index two). In particular, this is the case for the groups we encounter in this paper.

The symmetric genus problem has a rather extensive literature. When the finite group $G$ admits $(2,3,7)$-generation, that is, when $G$ can be generated by two of its elements $x$ and $y$ of respective orders 2 and 3 with product of order 7, we call $G$ a Hurwitz group. These groups have enormous intrinsic appeal (see, e.g., [5]). When $G$ is a Hurwitz group its (strong) symmetric genus is given by $1+|G| / 84$, an immediate consequence of the Riemann-Hurwitz equation [12]; so the symmetric genus problem is instantly solved for this family of groups, among whose members we find all but finitely many of the alternating

Received by the editors April 10, 1991.

1991 Mathematics Subject Classification. Primary 20D08; Secondary 57S25. 
groups [4], certain two-dimensional projective linear groups [16], certain Ree groups [20], and almost half of the sporadics [26] (cf. Table 6).

When $G$ is any finite group that fails to act on the sphere or the torus, $1+|G| / 84$ provides a lower bound on the strong symmetric genus of $G$, and for groups that are close to this bound (arguably all nonabelian simple groups), Tucker showed that $G$ must have at least one generating set of a given number of types [23]. This provides the modus operandi for calculating the symmetric genus of the groups in this paper.

For additional details see $[8,28]$. The article [23] is especially recommended. For treatises on the classical theory of Fuchsian groups, hyperbolic geometry, Riemann surfaces, and triangle groups-all of which have relevance to the symmetric genus problem-see [1, 10, 13, 17]. For additional information on Hurwitz groups see [5].

A word now about notation and terminology. Conjugate classes are denoted as in [9] (e.g., $2 A, 2 B, \ldots, 3 A, 3 B, \ldots$ ). We use $X, Y, Z$ (and on occasion $U, V, W)$ as variables (e.g., $2 X$ denotes a general class of involutions). We call a group $G(r X, s Y, t Z)$-generated provided there exist elements $x$, $y$ such that $G=\langle x, y\rangle, x \in r X, y \in s Y$, and $x y \in t Z$. In such cases $(r X, s Y, t Z)$ is referred to as a generating triple for $G$ and we call $G$ an $(r X, s Y, t Z)$-group. For $G$-classes $r X, s Y, t Z$ we denote by $\zeta(r X, s Y, t Z)$ the (unsymmetrized) structure constant of the group algebra $\mathbb{C} G$ corresponding to these classes. As is well known, $\zeta(r X, s Y, t Z)$ can be readily computed from the group character table (see [25], e.g.). When referring to a structure constant of a subgroup (or overgroup) $H$ of $G$, we specify $H$ in our notation $\zeta_{H}(r X, s Y, t Z)$. (When this is done, $r X, s Y$, and $t Z$ are understood to represent $H$-classes.) Similarly $C(r X)$ denotes the centralizer in $G$ of an element from the $G$-class $r X$, while the centralizer in a subgroup (or overgroup) $H$ of $G$ carries an identifying subscript $C_{H}(r X)$.

In closing, we remark that this research adds to an ever-increasing testimonial to the powerful influence of electronic mail on the resolution of mathematical problems; indeed only 1 of the $3=\left(\begin{array}{l}3 \\ 2\end{array}\right)$ pairs of authors has ever had the pleasure of meeting and, for the duration of this work, electronic mail provided the sole source of communication. One consequence of this is that the results in many cases were duplicated (even triplicated in some instances), and this was a constant source of delight for the authors as it provided frequent and welcomed confirmation.

\section{Preliminary Results}

In this section we discuss techniques that are useful in resolving generationtype questions for finite groups. We begin with two theorems that, in certain situations, are very effective at establishing nongeneration (see [21, 19]).

Theorem (L. Scott). Let $x_{1}, x_{2}, \ldots, x_{m}$ be elements generating a group $G$ with $x_{1} x_{2} \cdots x_{m}=1$, and let $V$ be an irreducible module for $G$ of dimension $n$. Let $C_{V}\left(x_{i}\right)$ denote the fixed point space of $\left\langle x_{i}\right\rangle$ on $V$, and let $d_{i}$ be the dimension of $V / C_{V}\left(x_{i}\right)$. Then $d_{1}+d_{2}+\cdots+d_{m} \geq 2 n$.

Theorem (Ree). Let $x_{1}, x_{2}, \ldots, x_{m}$ be permutations generating a transitive group on $n$ letters, with $x_{1} x_{2} \cdots x_{m}=1$, and let $c_{i}$ denote the number of orbits 
of $\left\langle x_{i}\right\rangle, 1 \leq i \leq m$. Then

$$
c_{1}+c_{2}+\cdots+c_{m} \leq(m-2) n+2 .
$$

We shall also frequently use the following lemmas:

Lemma 1. Let $G$ be $(r X, s Y, t Z)$-generated. Then for any overgroup $H$ of $G$ for which $C_{H}(G)=1$ we have $\zeta_{H}(r U, s V, t W) \geq\left|C_{H}(t W)\right|$, where $r U, s V$, $t W$ are the $H$-classes covering $r X, s Y, t Z$. In particular, $\zeta(r X, s Y, t Z) \geq$ $|C(t Z)|$ if $G$ is centerless.

Proof. Trivial.

Lemma 2. Let $G$ be simple and $(2 X, s Y, t Z)$-generated. Then $G$ is $(s Y, s Y$, $q W)$-generated, where $q W$ is the class into which $t Z$ squares.

Proof. Choose $x \in 2 X, y \in s Y$ with $G=\langle x, y\rangle$ and $z=x y \in t Z$. Clearly $\left\langle y^{x}, y\right\rangle$ is a normal subgroup of $G$, whence $\left\langle y^{x}, y\right\rangle=G$. Finally $y^{x} y=$ $x y x y=z^{2}$.

Perhaps the most powerful tool at one's disposal for settling issues of group generation is the computer system CAYLEY, developed by John Cannon [2]. We briefly explain one of the ways in which CAYLEY was employed in the current work. Suppose we are given the conjugate classes $K_{1}, K_{2}$, and $K_{3}$. We enumerate the elements $x$ of $K_{1}$ and then, for any fixed $z \in K_{3}$, we check to see whether $x^{-1} z \in K_{2}$. Those $x$ for which this is the case can then be partitioned into classes under conjugation by $C(z)$ and the order of the subgroup $\langle x, z\rangle$ computed. This is done until either all of the $\zeta\left(K_{1}, K_{2}, K_{3}\right)$ triples in the group have been accounted for, or until one such triple is found that generates the entire group. Although we shall not discuss them here, other algorithms were employed in our work (implemented on CAYLEY); details can be found in $[3,7]$. Some random searches for generating triples were also carried out using Richard Parker's Meat-Axe System [18].

\section{Main Results}

Proposition 1. The symmetric genus of $\mathbf{J}_{3}$ is 1255825 and arises from $(2,4,5)$ generation.

Proof. Explicit generation of type $(2,4,15)$ for $3 \mathrm{~J}_{3}$ was obtained via the Meat-axe using the 9-dimensional unitary representation for $3 \mathrm{~J}_{3}$ over $G F(4)$. Modulo multiplier, this established $(2 A, 4 A, 5 A)$-generation of $\mathrm{J}_{3}$. (Additionally, a nonconstructive proof of such generation was found by one of the authors by showing that the full structure constant $\zeta(2 A, 4 A, 5 A)$ could not be accounted for in proper subgroups of $\mathbf{J}_{3}$.) It suffices, therefore, to eliminate all generations of type $(r, s, t)$ where $r^{-1}+s^{-1}+t^{-1}>19 / 20$, i.e., those of type $(2,3,7)$ and $(2,3,8)$. The former case was treated in [24]. For the latter case, observe that $\zeta(2 A, 3 A, 8 A)=36$ and 32 such triples can be accounted for in the subgroup $\left(3 \times A_{6}\right): 2_{2}$. This leaves at most four available triples to generate $\mathrm{J}_{3}$, too few as $4<|C(8 A)|$. For the case $(2 A, 3 B, 8 A)$ apply Scott's Theorem to the 85-dimensional complex irreducible module $V$ for $\mathbf{J}_{3}$. (Note that $\operatorname{dim}\left(V / C_{V}(x)\right)=40, \operatorname{dim}\left(V / C_{V}(y)\right)=54, \operatorname{dim}\left(V / C_{V}(z)\right)=74$ where $x \in 2 A, y \in 3 B, z \in 8 A)$.

Proposition 2. The symmetric genus for $\mathrm{McL}$ is 78586201 and arises from $(2,5,8)$-generation. 


\begin{tabular}{crrrrrrrr}
\multicolumn{8}{c}{ TABLE 1} \\
$\qquad c l(x)$ & $2 A$ & $3 A$ & $3 B$ & $4 A$ & $5 B$ & $6 A$ & $6 B$ & $7 A$ \\
$\operatorname{dim}\left(V / C_{V}(x)\right)$ & 8 & 18 & 12 & 14 & 16 & 18 & 16 & 18
\end{tabular}

Proof. Explicit generation for McL of type $(2 A, 5 A, 8 A)$ was obtained via CAYLEY, using its 275-point permutation representation corresponding to the action of $\mathrm{McL}$ on the cosets of $U_{4}(3)$. It is therefore necessary to eliminate all generations of type $(r, s, t)$ where $r^{-1}+s^{-1}+t^{-1}>33 / 40$, as well as those of type $(2,2,2,3)$. In [24] this was done for generations of type $(2,3, t)$; the remaining cases are handled below. We first give the dimensions of the commutator spaces $V / C_{V}(x)$ for relevant $x$, where $V$ is the 22-dimensional complex irreducible module for McL (see Table 1).

Case $(2,4, t)$. Surely $\operatorname{dim}\left(V / C_{V}(z)\right)<22$ for any element $z$ of order $t$ in McL for which $1 / 2+1 / 4+1 / t>33 / 40$, so Scott's Theorem applies here.

Case $(3,3,4)$. Scott's Theorem eliminates $(3 B, 3 B, 4 A)$. Nongeneration follows from Lemma 1 since $\zeta(3 A, 3 B, 4 A)=0$ and $\zeta(3 A, 3 A, 4 A)=4<$ $96=|C(4 A)|$.

Case $(2,5,5)$. Scott's Theorem eliminates $(2 A, 5 B, 5 B)$. Nongeneration follows from Lemma 1 since $\zeta(2 A, 5 A, 5 A)=\zeta(2 A, 5 A, 5 B)=0$.

Case $(2,5,6)$. The triples $(2 A, 5 B, 6 A)$ and $(2 A, 5 B, 6 B)$ are eliminated by Scott's Theorem. Since $\zeta(2 A, 5 A, 6 A)=0$ and $\zeta(2 A, 5 A, 6 B)=36$, nongeneration follows - the latter by Lemma 1 as $\left|C_{A}(6 B)\right|=72$ for $A=$ Aut $(\mathrm{McL})$.

Case $(3,3,5)$. Scott's Theorem eliminates $(3 B, 3 B, 5 B)$. Nongeneration now follows from Lemma 1 since $\zeta(3 A, 3 A, 5 B)=10<25=|C(5 B)|$, $\zeta(3 A, 3 B, 5 A)=375<750=|C(5 A)|$ and all other structure constants are zero.

Case $(2,5,7)$. Scott's Theorem eliminates $(2 A, 5 B, 7 A)$. Lemma 1 eliminates $(2 A, 5 A, 7 A)$ as $\zeta(2 A, 5 A, 7 A)=7<14=|C(7 A)|$.

Case $(2,6,6)$. The triples $(2 A, 6 A, 6 B)$ and $(2 A, 6 B, 6 B)$ are eliminated by Scott's Theorem. Lemma 1 eliminates $(2 A, 6 A, 6 A)$ as $\zeta(2 A, 6 A, 6 A)$ $=240<360=|C(6 A)|$.

Case $(3,3,6)$. Scott's Theorem eliminates $(3 B, 3 B, 6 A)$ and $(3 B, 3 B, 6 B)$. As $\zeta(3 A, 3 A, 6 A)=15<360=|C(6 A)|, \zeta(3 A, 3 B, 6 B)=12<36=$ $|C(6 B)|$, and all remaining structure constants are 0 , the result follows.

Case $(3,4,4)$. Scott's Theorem eliminates $(3 B, 4 A, 4 A)$. Computing the relevant structure constant in $C=\mathrm{Co}_{3}$, we obtain $\zeta_{C}(3 A, 4 B, 4 B)=736<$ $1536=\left|C_{C}(4 B)\right|$, whence Lemma 1 eliminates $(3 A, 4 A, 4 A)$.

Case $(2,2,2,3)$. Both $(2 A, 2 A, 2 A, 3 A)$ and $(2 A, 2 A, 2 A, 3 B)$ are eliminated by Scott's Theorem.

Proposition 3. The symmetric genus of Suz is 11208637441 and arises from $(2,4,5)$-generation.

Proof. Explicit generation of type $(2,4,15)$ for $3 \mathrm{Suz}$ was obtained via the Meat-axe, using the 12-dimensional irreducible representation for 3Suz over $G F(4)$. Modulo center, this established $(2 B, 4 D, 5 B)$-generation of Suz . 
TABLE 2

$\begin{array}{ccccccccc}\operatorname{ccl}(x) & 2 A & 2 B & 3 A & 3 B & 3 C & 8 A & 8 B & 8 C \\ \operatorname{dim}\left(V / C_{V}(x)\right) & 64 & 72 & 72 & 90 & 96 & 120 & 122 & 124\end{array}$

Thus we must eliminate all generations of type $(r, s, t)$ for which $r^{-1}+s^{-1}+$ $t^{-1}>19 / 20$, viz. $(2,3,7)$ and $(2,3,8)$. The former was treated in [24]. For the latter, let $V$ denote the 143-dimensional irreducible module for Suz . The respective dimensions of the commutator spaces $V / C_{V}(x)$ are outlined in Table 2.

Thus Scott's Theorem eliminates all types of $(2,3,8)$-generation with the exception of $(2 B, 3 C, 8 A),(2 B, 3 C, 8 B),(2 B, 3 C, 8 C)$, and $(2 B, 3 B, 8 C)$.

Case $(2 B, 3 C, 8 A)$. We claim that, of the full structure constant $\zeta(2 B, 3 C, 8 A)=1920$, at least 1728 can be accounted for in $G_{2}(4)$ subgroups of Suz. To see this observe that each $G_{2}(4)$ is a point stabilizer in the action of Suz on the Suzuki graph [9] and that the two-point stabilizers are $\mathbf{J}_{2}$ and $2^{2+8}:\left(A_{5} \times 3\right)$. Trivially, $e \in 8 A$ lies in six conjugate $G_{2}(4)$ subgroups and, by analysis of centralizer orders, precisely 12 of the 15 pairwise intersections of these conjugates yields a $\mathbf{J}_{2}$. If $K$ is a $(2,3,8)$-subgroup of $2^{2+8}:\left(A_{5} \times 3\right)$, then $K$ surely embeds in $2^{2+8}: A_{5}$. But elements of order 3 in $2^{2+8}: A_{5}$ must centralize an involution in the Sylow-central $2^{2}$, which is $2 A$-pure. This shows that $K$ is not $(2 B, 3 C, 8 A)$-generated, whence a total contribution of at least

$$
6 \zeta_{G}-12 \zeta_{J}=6(416)-12(64)=1728
$$

results from triples within $G_{2}(4)$ subgroups, as claimed. (Here $\zeta_{G}$ and $\zeta_{J}$ denote the relevant structure constants for the groups $G=G_{2}(4)$ and $J=J_{2}$.) This leaves at most 192 triplies, too few for any to generate Suz as $\left|C_{A}(8 A)\right|=$ 384 for $A=\operatorname{Aut}(\mathrm{Suz})$.

Case $(2 B, 3 C, 8 B)$. Here we found it easier to work with $(2 B, 8 B, 3 C)$ triples of which there are $\zeta(2 B, 8 B, 3 C)=64800$. Using CAYLEY we were able to account for all triples as follows: 25920 of them generated subgroups of order 124800 (there are $8 C(3 C)$-classes of such triples, each of size 3240), 25920 of them generate subgroups of order 4032 (again $8 C(3 C)$ )-classes of size 3240 each), and 12960 of them generate subgroups of order 96 (4C(3C)-classes of size 3240 each).

Case $(2 B, 3 C, 8 C)$. Again we found it easier to work with $(2 B, 8 C, 3 C)$ triples of which there are $\zeta(2 B, 8 C, 3 C)=81000$. Using CAYLEY we discovered that 19440 of these triples generate subgroups of order 11232 (six $C(3 C)$-classes of size 3240 each), 12960 generate subgroups of order 4032 (four $C(3 C)$-classes of size 3240 each), 25920 generate subgroups of order 1008 (eight $C(3 C)$-classes of size 3240 each), 6480 generate subgroups of order 336 (four $C(3 C)$-classes of size 1620 each), and 16200 generate subgroups of order 96 (seven $C(3 C)$-classes in all, four of which have size 1620 and three of which have size 3240 ).

Case $(2 B, 3 B, 8 C)$. If Suz were $(2 B, 3 B, 8 C)$-generated then it would have to be $(3 B, 3 B, 4 C)$-generated as well (Lemma 2$)$. But the latter generation 
is impossible by Lemma 1 as $\zeta_{A}(3 B, 3 B, 4 C)=1728<3072=\left|C_{A}(4 C)\right|$ for $A=\operatorname{Aut}(\mathrm{Suz})$.

Proposition 4. The symmetric genus of $\mathrm{O}^{\prime} \mathrm{N}$ is 9600323041 and arises from $(2,3,8)$ generation.

Proof. Explicit generation of type $(2,3,8)$ for $3^{\prime} \mathrm{O}$ N was obtained via the Meat-axe, using its 45-dimensional complex irreducible representation. Modulo multiplier, this established $(2 A, 3 A, 8 A)$-generation of $\mathrm{O}^{\prime} \mathrm{N}$. Thus one need only eliminate $(r, s, t)$-generation for which $r^{-1}+s^{-1}+t^{-1}>23 / 24$, i.e., $(r, s, t)=(2,3,7)$. And this was achieved in [24].

Proposition 5. The symmetric genus of $\mathrm{Co}_{2}$ is 1602478080001 and arises from $(2,3,11)$-generation.

Proof. That $\mathrm{Co}_{2}$ is $(2,3,11)$-generated was proved in [26]. It therefore suffices to eliminate all generations $(r, s, t)$ for which $r^{-1}+s^{-1}+t^{-1}>61 / 66$, viz. $(2,3, t), 7 \leq t \leq 10$, and $(2,4,5)$. The case $(2,3,7)$ was treated in [24]. Before handling the remaining cases, we list (Tables 3 and 4$)$ the dimensions of the commutator spaces $V / C_{V}(x)$ and $W / C_{W}(x)$, where $V$ and $W$ denote, respectively, the 23- and 275-dimensional complex irreducible modules for $\mathrm{Co}_{2}$. This is followed by a listing (Table 5) of the number of orbits of $\langle x\rangle$ in the action of $\mathrm{Co}_{2}$ on the rank 3 graph on 2300 points [9].

Case $(2,3,8)$. Except for $(2 B, 3 A, 8 F)$, Ree's Theorem eliminates all generations of type $(2 A, 3 Y, 8 Z)$ and $(2 B, 3 Y, 8 Z)$ (see Table 5). Except for $(2 C, 3 B, 8 D)$, Scott's Theorem eliminates all generations of type $(2 C, 3 B, 8 Z)$ (see Table 3). Finally, except for $(2 C, 3 A, 8 F)$, Lemma 1 eliminates all generations of type $(2 C, 3 A, 8 Z)$ as $\zeta(2 C, 3 A, 8 Z)<|C(8 Z)|$ for every $Z \neq F$. So we must treat the surviving cases $(2 B, 3 A, 8 F),(2 C, 3 B, 8 D)$, and $(2 C, 3 A, 8 F)$. But the first of these is handled by Scott's Theorem (Table 3) while Lemma 1 eliminates the second, since $\zeta(2 C, 3 B, 8 D)=192<$ $512=|C(8 D)|$. Now suppose $\mathrm{Co}_{2}$ is $(2 C, 3 A, 8 F)$-generated. By Lemma $2, \mathrm{Co}_{2}$ must also be $(3 A, 3 A, 4 E)$-generated, whence $C=\mathrm{Co}_{1}$ must contain a $(3 C, 3 C, 4 C)$-subgroup $K$ isomorphic to $\mathrm{Co}_{2}$. But, as $C_{C}(K)=1$ and $\zeta_{C}(3 C, 3 C, 4 C)=251904<737280=\left|C_{C}(4 C)\right|$, this contradicts Lemma 1. We conclude that $\mathrm{Co}_{2}$ is not $(2,3,8)$-generated.

Case $(2,4,5)$. We consider in succession triples of type $(2 A, 4 Y, 5 Z)$, $(2 B, 4 Y, 5 Z)$, and $(2 C, 4 Y, 5 Z)$. Observe that Ree's Theorem eliminates all generations of the first type (see Table 5). For the triples $(2 B, 4 Y, 5 Z)$, Scott's Theorem (Table 3) eliminates all $(2 B, 4 Y, 5 B)$-generations as well as those of type $(2 B, 4 A, 5 A),(2 B, 4 C, 5 A),(2 B, 4 E, 5 A)$, and $(2 B, 4 F, 5 A)$. Ree's Theorem now rules out $(2 B, 4 B, 5 A)$, and $(2 B, 4 D, 5 A)$, while Scott's Theorem, applied to the module $W$ (Table 4), handles $(2 B, 4 G, 5 A)$. We turn finally to the triples $(2 C, 4 Y, 5 Z)$. Except for $(2 C, 4 G, 5 A)$ and $(2 C, 4 G$, $5 B$ ), these are all successfully handled by Scott's Theorem (see Table 3 for the types $(2 C, 4 A, 5 B),(2 C, 4 C, 5 B),(2 C, 4 E, 5 B),(2 C, 4 F, 5 B)$ and Table 4 for all others). For $(2 C, 4 G, 5 A)$ we observe that $\zeta(2 C, 4 G, 5 A)=$ 15000 and that all such triples can be accounted for as follows: 12000 generate subgroups of order 737280 (eight $C(5 A)$-classes of 1500 each), 2250 generate subgroups of order 1920 (three $C(5 A)$-classes of 750 each), 750 generate subgroups of order 120 (a unique $C(5 A)$-class). Finally $\zeta(2 C, 4 G, 5 B)=18000$, 
TABLE 3. Dimensions of the commutator spaces $V / C_{V}(x)$ where $V$ denotes the 23-dimensional irreducible module for $\mathrm{Co}_{2}$.

$\begin{array}{ccccccccccc}\operatorname{ccl}(x) & 2 B & 2 C & 3 A & 3 B & 4 A & 4 C & 4 E & 4 F & 5 A & 5 B \\ \operatorname{dim}(V / C(x)) & 8 & 12 & 18 & 12 & 16 & 14 & 14 & 16 & 20 & 16 \\ & & & & & & & & & & \\ \operatorname{ccl}(x) & 8 A & 8 B & 8 C & 8 E & 8 F & 9 A & 10 A & 10 B & 10 C & \\ \operatorname{dim}\left(V / C_{V}(x)\right) & 20 & 20 & 20 & 18 & 18 & 20 & 20 & 20 & 20 & \end{array}$

TABLE 4. Dimensions of the commutator spaces $W / C_{W}(x)$ where $W$ denotes the 275-dimensional irreducible module for $\mathrm{Co}_{2}$.

$\begin{array}{ccccccccc}c c l(x) & 2 B & 2 C & 3 A & 4 A & 4 B & 4 C & 4 D & 4 E \\ \operatorname{dim}\left(W / C_{W}(x)\right) & 120 & 132 & 180 & 184 & 190 & 194 & 196 & 194 \\ & & & & & & & & \\ c c l(x) & 4 F & 4 G & 5 A & 5 B & 9 A & 10 A & 10 B & 10 C \\ \operatorname{dim}\left(W / C_{W}(x)\right) & 196 & 204 & 220 & 216 & 242 & 244 & 240 & 244\end{array}$

TABLE 5. Number of orbits of $\langle x\rangle$ in the action of $\mathrm{Co}_{2}$ on the rank 3 graph on 2300 points.

$\begin{array}{cccccccccc}c c l(x) & 2 A & 2 B & 3 A & 3 B & 4 A & 4 B & 4 C & 4 D & 4 E \\ \text { \# orbits } & 1292 & 1220 & 770 & 794 & 660 & 630 & 626 & 648 & 618 \\ & & & & & & & & & \\ c c l(x) & 4 F & 4 G & 5 A & 5 B & 8 A & 8 B & 8 C & & \\ \text { \# orbits } & 616 & 588 & 460 & 468 & 332 & 318 & 326 & & \\ & & & & & & & & \\ \operatorname{ccl}(x) & 8 D & 8 E & 8 F & 9 A & 10 A & 10 B & & & \\ \text { \# orbits } & 324 & 316 & 310 & 258 & 244 & 260 & & & \end{array}$

and these triples are accounted for as follows: 5400 generate subgroups of order 88704000 (nine $C(5 B)$-classes of 600 each), 3600 generate subgroups of order 1351680 (six $C(5 B)$-classes of 600 each), 4800 generate subgroups of order 368640 (eight $C(5 B)$-classes of 600 each), 2400 generate subgroups of order 5120 (eight $C(5 B)$-classes of 300 each), 600 generate subgroups of order 600 (a unique $C(5 B)$-class), 1200 generate subgroups of order 160 (four $C(5 B)$-classes of size 300 each).

Case $(2,3,9)$. Ree's Theorem eliminates the types $(2 A, 3 Y, 9 A)$. Scott's Theorem eliminates $(2 B, 3 B, 9 A),(2 C, 3 B, 9 A)$ (Table 3$)$ and $(2 B, 3 A, 9 A)$ (Table 2). This leaves only $(2 C, 3 A, 9 A)$ for which one computes $\zeta(2 C, 3 A, 9 A)=108$. Using CAYLEY we were able to account for the full structure constant as follows: 81 triples generate a group of order 258048 (three 
$C(9 A)$-classes of size 27 each), while the remaining 27 triples generate a group of order 504. (In this case it is not hard to identify these groups. They are $2^{1+8}: L_{2}(8)$ and $L_{2}(8)$.) So $\mathrm{Co}_{2}$ is not $(2,3,9)$-generated.

Case $(2,3,10)$. We consider in succession triples of type $(2 A, 3 Y, 10 Z)$, $(2 B, 3 Y, 10 Z)$, and $(2 C, 3 Y, 10 Z)$. Ree's Theorem eliminates generations of the first type except for $(2 A, 3 A, 10 C)$, which can be ruled out by application of Scott's Theorem to the 253-dimensional complex irreducible module $U: \operatorname{dim}\left(U / C_{U}(x)\right)=112, \operatorname{dim}\left(U / C_{U}(y)\right)=162, \operatorname{dim}\left(U / C_{U}(z)\right)=225$ $(x \in 2 A, y \in 3 A, z \in 10 C)$. All $(2 B, 3 Y, 10 Z)$-generations are ruled out by Scott's Theorem: Use Table 4 for the case $Y=A$ and Table 3 for the case $Y=B$. Finally Scott's Theorem (Table 3 ) handles the triples $(2 C, 3 B, 10 Z)$,

TABLE 6. Symmetric genus and corresponding generating triples for 23 of the 26 sporadic groups. For $\mathrm{Fi}_{23}, \mathrm{~B}$ and $\mathrm{M}$, the problem is as yet unresolved.

\begin{tabular}{|c|c|c|c|}
\hline$G$ & symmetric genus of $G$ & $(r, s, t)$ & Proof \\
\hline $\mathbf{M}_{11}$ & 631 & $(2,4,11)$ & {$[25,6]$} \\
\hline $\mathbf{M}_{12}$ & 3169 & $(2,3,10)$ & {$[25,6]$} \\
\hline $\mathrm{J}_{1}$ & 2091 & $(2,3,7)$ & [20] \\
\hline $\mathbf{M}_{22}$ & 34849 & $(2,5,7)$ & {$[25,6]$} \\
\hline $\mathbf{J}_{2}$ & 7201 & $(2,3,7)$ & [11] \\
\hline $\mathrm{M}_{23}$ & 1053361 & $(2,4,23)$ & [6] \\
\hline HS & 1680001 & $(2,3,11)$ & [27] \\
\hline $\mathbf{J}_{3}$ & 1255825 & $(2,4,5)$ & Proposition 1 \\
\hline $\mathrm{M}_{24}$ & 10200961 & $(3,3,4)$ & [6] \\
\hline McL & 78586201 & $(2,5,8)$ & Proposition 2 \\
\hline $\mathrm{He}$ & 47980801 & $(2,3,7)$ & [24] \\
\hline $\mathrm{Ru}$ & 1737216001 & $(2,3,7)$ & {$[24]$} \\
\hline Suz & 11208637441 & $(2,4,5)$ & Proposition 3 \\
\hline $\mathrm{O}^{\prime} \mathrm{N}$ & 9600323041 & $(2,3,8)$ & Proposition 4 \\
\hline $\mathrm{Co}_{3}$ & 5901984001 & $(2,3,7)$ & {$[29,24]$} \\
\hline $\mathrm{Co}_{2}$ & 1602478080001 & $(2,3,11)$ & Proposition 5 \\
\hline $\mathrm{Fi}_{22}$ & 768592281621 & $(2,3,7)$ & [26] \\
\hline $\mathrm{HN}$ & 3250368000001 & $(2,3,7)$ & [24] \\
\hline Ly & 616252131000001 & $(2,3,7)$ & [24] \\
\hline Th & 1080308855808001 & $(2,3,7)$ & [14] \\
\hline $\mathrm{Co}_{1}$ & 86620350136320001 & $(2,3,8)$ & Proposition 6 \\
\hline $\mathrm{J}_{4}$ & 1033042512453304321 & $(2,3,7)$ & [26] \\
\hline $\mathrm{Fi}_{24}^{\prime}$ & 14942925109412639539201 & $(2,3,7)$ & {$[15]$} \\
\hline
\end{tabular}


leaving only those of type $(2 C, 3 A, 10 Z)$. We compute $\zeta(2 C, 3 A, 10 Z)=$ 0 (for $Z=A$ ), 120 (for $Z=B$ ), and 80 (for $Z=C$ ). In the case of ( $2 C, 3 A, 10 B)$ the full structure constant is accounted for as follows: 90 triples generate subgroups of order 184320 (three $C(10 B)$-classes of size 30 each), while the remaining 30 triples generate subgroups of order 360 (explicitly $A_{5} \times$ $S_{3}$ ). Moving to $(2 C, 3 A, 10 C)$, we have that 60 triples (three $C(10 C)$-classes of size 20 each) generate $A_{5} \times S_{3}$. This concludes the proof that $\mathrm{Co}_{2}$ is not $(2,3,10)$-generated, hence Proposition 5 .

Proposition 6. The symmetric genus of $\mathrm{Co}_{1}$ is 86620350136320001 and arises from $(2,3,8)$-generation.

Proof. Explicit generation of type $(2,3,8)$ for $2 \mathrm{Co}_{1}$ was obtained via the Meat-axe, using its 24-dimensional complex irreducible representation. Modulo multiplier, this established $\left(2\right.$ ? $3 \mathrm{D}, 8$ ?)-generation of $\mathrm{Co}_{1}$ (the 2-class and 8class not completely determined). As in the case of $\mathrm{O}^{\prime} \mathrm{N}$, one must eliminate only $(2,3,7)$-generation, and this was done in [24].

We can now state our main result.

Theorem. For $G$ a sporadic simple group other than $\mathrm{Fi}_{23}, \mathrm{~B}$, or $\mathrm{M}$, the symmetric genus of $G$ is as indicated in Table 6.

Corollary. Let $G$ be a sporadic simple group and let $S$ be any (orientable) minimal genus surface on which $G$ acts in an orientation-preserving manner. Then $\operatorname{Aut}(S) \cong G$, where $\operatorname{Aut}(S)$ is the group of all orientation-preserving automorphisms of $S$.

Proof. This was established for all sporadic groups other than McL in [24]. As a minimal genus surface for $\mathrm{McL}$ is now known to arise from $(2,5,8)$-generation of $\mathrm{McL}$, the result follows from a classification of Singerman [22].

\section{Partial Results of $F_{23}$}

Proposition 7. The symmetric genus of $\mathrm{Fi}_{23}$ arises from one of the following generations: $(2,3,8),(2,4,5),(2,3,9)$. Among these, $\mathrm{Fi}_{23}$ is known to be $(2 C, 3 D, 9 E)$-generated. If $\mathrm{Fi}_{23}$ is $(2 X, 3 Y, 8 Z)$-generated then $(2 X, 3 Y$, $8 Z)$ is one of $(2 C, 3 D, 8 A),(2 C, 3 D, 8 B),(2 C, 3 D, 8 C)$. If $\mathrm{Fi}_{23}$ is $(2 X$, $4 Y, 5 Z)$-generated then $(2 X, 4 Y, 5 Z)$ is $(2 C, 4 C, 5 A)$ or $(2 C, 4 D, 5 A)$.

Proof. Explicit generation of type $(2 C, 3 D, 9 E)$ for $\mathrm{Fi}_{23}$ was obtained via the Meat-axe, using its 253-dimensional representation over $G F(3)$.

Case $(2,3,8)$. Applying Ree's Theorem to the permutation module arising from the action of $\mathrm{Fi}_{23}$ on its 31671 involutions of class $2 A$, we can eliminate all $(2 A, 3 Y, 8 Z)$-generations. The types $(2 C, 3 A, 8 Z)$ and $(2 C, 3 C, 8 C)$ are ruled out by Lemma 1 . By Lemma 2 generation of type either $(2 C, 3 C, 8 A)$ or $(2 C, 3 C, 8 B)$ implies $(3 C, 3 C, 4 B)$-generation of $\mathrm{Fi}_{23}$, which is impossible by Lemma 1 as $\zeta_{F}(3 C, 3 C, 4 A)<\left|C_{F}(4 A)\right|$ for $F=\mathrm{Fi}_{24}^{\prime}$. Finally Scott's Theorem applied to the 782-dimensional complex irreducible module of $\mathrm{Fi}_{23}$ eliminates all remaining generations except those mentioned in the statement of proposition.

Case $(2,4,5)$. Scott's Theorem on the aforementioned 782-dimensional module eliminates all generations except those of type $(2 C, 4 Y, 5 A)$. Of these, 
$(2 C, 4 A, 5 A)$ and $(2 C, 4 B, 5 A)$ are ruled out by Lemma 1 , as $\zeta_{\mathrm{B}}(2 D, 4 D, 5 A)$ and $\zeta_{\mathrm{B}}(2 D, 4 E, 5 A)$ are each strictly less than $\left|C_{\mathrm{B}}(5 A)\right|$ for $\mathrm{B}$ the Baby Monster. Thus we have eliminated all generations except the two mentioned in the statement of the proposition.

\section{REFERENCES}

1. A. F. Beardon, The geometry of discrete groups, Springer-Verlag, Berlin, 1980.

2. J. J. Cannon, An introduction to the group theory language CAYLEY, Computational Group Theory (M. Atkinson, ed.), Academic Press, San Diego and London, 1984, pp. 145-183.

3. M. D. E. Conder, Some results on quotients of triangule groups, Bull. Austral. Math. Soc. 30 (1984), 73-90.

4. _ The symmetric genus of alternating and symmetric groups, J. Combin. Theory Ser. B 39 (1985), 179-186.

5. __ Hurwitz groups: a brief survey, Bull. Amer. Math. Soc. (N.S.) 23 (1990), 359-370.

6. __ The symmetric genus of the Mathieu groups, Bull. London Math. Soc. 23 (1991), 445-453.

7. __ Random walks in large finite groups, Austral. J. Combin. 4 (1991), 49-57.

8. M. D. E. Conder, R. A. Wilson, and A. J. Woldar, The symmetric genus of sporadic groups: announced results (submitted).

9. J. H. Conway, R. T. Curtis, S. P. Norton, R. A. Parker, and R. A. Wilson, ATLAS of finite groups, Clarendon Press, Oxford, 1985.

10. H. S. M. Coxeter and W. O. J. Moser, Generators and relations for discrete groups, 4th ed., Springer-Verlag, Berlin, 1980.

11. L. Finkelstein and A. Rudvalis, Maximal subgroups of the Hall-Janko-Wales group, J. Algebra 24 (1973), 486-493.

12. A. Hurwitz, Über algebraische Gebilde mit eindeutigen Transformationen in sich, Math. Ann. 41 (1893), 403-442.

13. G. A. Jones and D. Singerman, Complex functions: an algebraic and geometric viewpoint, Cambridge Univ. Press, Cambridge, 1987.

14. S. A. Linton, The maximal subgroups of the Thompson group, J. London Math. Soc. 39 (1989), 79-88.

15. S. A. Linton and R. A. Wilson, The maximal subgroups of the Fischer groups $\mathrm{Fi}_{24}^{\prime}$ and $\mathrm{Fi}_{24}$, Proc. London Math. Soc. 63 (1991), 113-164.

16. A. M. Macbeath, Generators of the linear fractional groups, Number Theory, Proc. Sympos. Pure Math., vol 12, Amer. Math. Soc., Providence, RI, 1969, pp. 14-32.

17. W. Magnus, Noneuclidean tesselations and their groups, Academic Press, New York, 1974.

18. R. A. Parker, Computer calculation of modular characters-the Meat-axe, Computational Group Theory (M. Atkinson, ed.), Academic Press, San Diego and London, 1984, pp. 267-274.

19. R. Ree, A theorem on permutations, J. Combin. Theory Ser. A 10 (1971), 174-175.

20. C.-H. Sah, Groups related to compact Riemann surfaces, Acta Math. 123 (1969), 13-42.

21. L. L. Scott, Matrices and cohomology, Ann. of Math. (2) 105 (1977), 473-492.

22. D. Singerman, Finitely maximal Fuchsian groups, J. London Math. Soc. (2) 6 (1972), 2938.

23. T. W. Tucker, Finite groups acting on surfaces and the genus of a group, J. Combin. Theory Ser. B 34 (1983), 82-98.

24. A. J. Woldar, On Hurwitz generation and genus actions of sporadic groups, Illinois J. Math. 33 (1989), 416-437.

25. _ Representing $\mathbf{M}_{i i}, \mathbf{M}_{12}, \mathrm{M}_{22}$ and $\mathrm{M}_{23}$ on surfaces of least genus, Comm. Algebra 18 (1990), 15-86. 
26. __ Sporadic simple groups which are Hurwitz, J. Algebra 144 (1991), 443-450.

27. _ The symmetric genus of the Higman-Sims group HS and bounds for Conway's groups $\mathrm{Co}_{1}, \mathrm{Co}_{2}$, Illinois J. Math. 36 (1992), 47-52.

28. _ On the symmetric genus of simple groups (submitted).

29. M. F. Worboys, Generators for the sporadic group $\mathrm{Co}_{3}$ as a $(2,3,7)$-group, Proc. Edinburgh Math. Soc. (2) 25 (1982), 65-68.

Department of Mathematics and Statistics, The University of Auckland, Auckland, New ZeALAND

E-mail address: conder@maths.aukuni.ac.nz

School of Mathematics and Statistics, The University of Birmingham, BirmingHAM, ENGLAND

E-mail address: wilsonra@ibm3090.bham.ac.uk

Department of Mathematics Sciences, Villanova University, Villanova, PennsylVANIA 19085

E-mail address: woldar@ucis.vill.edu 